\title{
Narrowing the Duane syndrome critical region at chromosome 8q13 down to $40 \mathrm{~kb}$
}

\author{
G Calabrese ${ }^{1}$, L Telvi $^{2}$, F Capodiferro $^{1}$, E Morizio $^{1}$, A Pizzuti $^{3}$, L Stuppia ${ }^{1,4}$, R Bordoni $^{3}$, \\ A Ion ${ }^{2}$, D Fantasia ${ }^{1}$, R Mingarelli $^{5}$ and G Palka ${ }^{1}$ \\ ${ }^{1}$ Dipartimento di Scienze Biomediche/Sez. Genetica Medica, Università di Chieti, Italy; ${ }^{2}$ Laboratoire de \\ Cytogénétique, Hôpital Saint Vincent De Paul, Paris, France; ${ }^{3}$ Istituto di Clinical Neurologica, Università di Milano; \\ ${ }^{4}$ Istituto di Citomorfologia Umana Normale e Patologica del CNR, Chieti; ${ }^{5}$ Mendel-CSS IRCSS, Rome, Italy
}

Duane syndrome (M IM 126800) is an autosomal dominant disorder characterised by primary strabismus and other ocular anomalies, associated with variable deficiency of binocular sight. We have recently identified a <3cM smallest region of deletion overlap (SRO) by comparing interstitial deletions at band 8q13 in two patients (one described by Vincent et al, 1994, and the other by Calabrese et al, 1998). Here we report on another patient with Duane syndrome carrying a reciprocal translation $t(6 ; 8)(q 26 ; q 13)$. FISH and PCR analyses using a YAC contig spanning the SRO narrowed the Duane region to a $<1 \mathrm{cM}$ interval between markers SHGC37325 and W14901. In addition, the identification and mapping of two PAC clones flanking the translocation breakpoint, allowed us to further narrow the critical region to about $40 \mathrm{~kb}$. As part of these mapping studies, we have also refined the map position of AMYB, a putative candidate gene, to $8 q 13$, centromeric to Duane locus. AMYB is expressed in brain cortex and genital crests and has been previously mapped to 8q22. European Journal of Human Genetics (2000) 8, 319-324.

Keywords: Duane syndrome; 8q13; physical mapping

\section{Introduction}

Duane syndrome (MIM 126800) ${ }^{1}$ is an autosomal dominant syndrome with unknown etiology. It is responsible for a primary form of strabismus, accompanied by bilateral globe retraction and narrowing of the palpebral fissure, leading to deficiencies of binocular sight of variable severity. ${ }^{2}$ The pathogenesis of this syndrome is unknown but, in some patients a muscular-neuronal origin has been proven. $^{3}$ Although mostly reported as an isolated disorder, in a few patients Duane syndrome was associated with deafness, renal defects, muscular and skeletal anomalies, and genetic heterogeneity has even been described. ${ }^{4}$ Furthermore, chromosome anomalies have been observed in some Duane patients and an 8q12-13 contiguous gene syndrome including Duane syndrome has been proposed. ${ }^{5}$ Recently, a comparison of boundaries in two patients with $8 q 13$ deletions $s^{5,6}$ allowed us

Correspondence: Giandomenico Palka MD, Via B. Buozzi 93-65121 Pescara, Italy. Tel: +390871 3554 136; Fax: + 3908713554 135; E-mail: gdpalka@unich.it

Received 5 July 1999; revised 3 December 1999; accepted 9 December 1999 to narrow down the Duane syndrome smallest region of overlap (SRO) to a $<3 \mathrm{CM}$ interval between D8S533 and D8S1767. ${ }^{6}$

In this study we report a Duane syndrome patient carrying a constitutional translocation involving region 8q13. Molecular analyses have allowed the refinement of the Duane locus, and the reassignment of the map position of the AMYB gene, proposed as a candidate gene due its expression in the hindbrain, neural retina and urogenital ridge. ${ }^{7}$

\section{Materials and methods Patient}

A 31-year-old man was referred for cytogenetic analysis because of infertility. On clinical evaluation he presented with hypoplastic external genitalia and glandular hypospadias. Additional clinical features included strabismus with amblyopia and narrowing of pal pebral fissures which led to a diagnosis of Duane syndrome. Routine laboratory investigations, echocardiography, renal ecography and audiometry 
were normal. Histological and endocrinological examinations reveal ed dysgenetic gonads.

\section{Cytogenetic and FISH analyses}

Chromosome studies were carried out on PHA-stimulated peripheral blood lymphocytes using high resolution GTG and RBG banding techniques.

FISH and fibre-FISH analyses were performed according to Cal abrese et $\mathrm{al}^{6}$ and Fidlerova et al, ${ }^{8}$ respectively. Biotinylated and FITC-labelled painting probes for chromosomes 6 and 8 (Cambio-Bouty, Italy), biotinylated 6q25-specific band probe (Li-Star FISH, Italy), YAC clones from contig WC8.8 (Généthon, France/Whitehead Institute, MIT, USA), PAC clones from RPCI-5 library (IGeR, Italy) were used. Probes were labelled by nick-translation prior to hybridisation.

\section{Sequence tagged sites (STS) analysis}

Fourteen STSS mapping within the Duane SRO at $8 q 13^{6}$ and retrieved from CEPH/Généthon database ${ }^{9}$ were tested by PCR on YAC clones to confirm integrity and on the PAC library for screening. Computer-aided search for sequences from genes and STS-ESTs mapped in the $8 q 13$ region was performed using software available at the Whitehead Institute and Genome DataBase websites. ${ }^{10,11}$

\section{Chromosome microdissection}

Microdissection was carried out according to Zhang protocols ${ }^{12}$ using a Nikon Eclipse inverted microscope and Nikon micromanipulator. Briefly, PCR using degenerated oligos ${ }^{13}$ was performed on 40 copies of chromosomes $\operatorname{der}(6)$ and der(8) by a three-step amplification procedure. PCR products were then tested for content of STS markers from the SRO.

\section{Results \\ Cytogenetic and YAC-based FISH characterisation of the 8q breakpoint}

GTG banding in the patient revealed a reciprocal translocation $\mathrm{t}(6 ; 8)(\mathrm{q} 26 ; \mathrm{q13})$ (Figure1a) which was confirmed by FISH experiments with chromosome 6 and 8 specific paintings.

In order to characterise chromosome8 breakpoint, six YAC clones mapping with in the SRO previously identified (759A7, 953G3, 761H11, 897B11, 820E6 and 910F5) ${ }^{6}$ were chosen. FISH analysis revealed that the four most centromeric clones 759A7, 953G3,761H11, and 897B11 maintained the normal position at 8q13 on der(8). Clone820E6 spanned the translocation $8 \mathrm{q}$ breakpoint as a large signal on normal chromosome8, and one signal each on $\operatorname{der}(8)$ and $\operatorname{der}(6)$ (Figure1b). Clone910F5 showed a signal on $\operatorname{der}(6)$ in addition to that on the normal chromosome8. To further refine the SRO, ten more YAC clones flanking 820E6 were chosen from the contig (Figure2). Six clones, proximal to 820E6 (816D4 to 809G3) and sharing marker AFM 238xc3, showed a normal location on der(8). The other four clones (925D9, $750 \mathrm{C} 10,883 \mathrm{~F} 2$ and 743G1) were partially overlapping with
clone820E6 more centromeric markers WI4901 to D8S1775. Three of these clones (925D9, 750C10, and 883F2) spanned the translocation breakpoint. In particular, clone925D9, in addition to a signal on normal chromosome8, produced a large signal on der(8) and a tiny signal on $\operatorname{der}(6)$, whileclones $750 \mathrm{C} 10$ and 883F2 displayed on $\operatorname{der}(6)$ a signal larger than the one on $\operatorname{der}(8)$. The remaining clone $743 \mathrm{G} 1$ did not span the translocation.

\section{STS analysis}

STS content of YACs ${ }^{10}$ used for FISH was confirmed by PCR analysis. Microdissected $\operatorname{der}(6)$ and $\operatorname{der}(8)$ products were also analysed by PCR using six STS markers (WI7816, WI6151, AFM 238xc3, WI4901, D8S1767, D8S1775) from the critical region. STS analysis showed that chromosome 8 DNA microdissected from der(8) was positive for markers WI7816 to AFM 238xc3 (located proximally within the SRO), while DNA from der(6) was positive for STSs WI4901 to D8S1775 (located distally within the SRO) (data not shown). Marker AFM 238xc3 was used to screen a PAC library but gave no positive clones, while WI4901 identified clone1172j16. While these studies were in progress, a new STS marker, SHGC37325, has been placed between AFM238xc3 and WI4901 by radiation hybrid mapping. ${ }^{11}$ By PCR analysis we found SHGC37325 in YAC clone925D9. Because this is the most proximal clone spanning the $8 \mathrm{q}$ breakpoint, we proceeded with PAC library screening using this STS and identified a new clone, $832 \mathrm{~m} 7$. FISH experiments with the two PAC clones showed that clone832m7 maps on der(8) and $1172 \mathrm{j} 16$ on der(6) (Figure3a). To understand the physical distance between the two clones $1172 \mathrm{j} 16$ and $832 \mathrm{m7}$, we performed fibre-FISH experiments that revealed a $\leq 40 \mathrm{~kb}$ gap between the two clones (Figure 3b).

A PAC library screening was also performed using ESTs WI7886 and WI7816, located at the $5^{\prime}$ and $3^{\prime}$ UTR, respectively of the AMYB gene. ${ }^{10,11}$ AMYB has been proposed as a putative can didate gene for Duane syndrome. A positive PAC clone was obtained, 889el5, and mapped by FISH to chromosome $8 q 13$ and on $\operatorname{der}(8)$ (Figure3c).

\section{Discussion}

We have previously proposed a SRO for Duane syndrome included between markers D8S533 and D8S1767 which harbours a $3 \mathrm{cM}$ region located between the loci for Freidreich ataxia with vitamin E deficiency and the Branchio-Oto-Renal syndrome gene EYA1 at $8 \mathrm{q} 13 .{ }^{6}$

In the present study we have described a patient with gonadal dysgenesis and Duane syndrome heterozygous for a translocation involving 8q13. Cytogenetic evidence for a reciprocal translocation $\mathrm{t}(6 ; 8)(\mathrm{q} 26 ; \mathrm{q} 13)$ was confirmed by FISH analysis using a panel of 16YAC clones from a $8 q 13$ contig. In addition, in situ hybridisation localised the $8 q$ breakpoint in a region between markers AFM 238xc3 and WI4901. The location of the translocation breakpoint on $8 q$ 


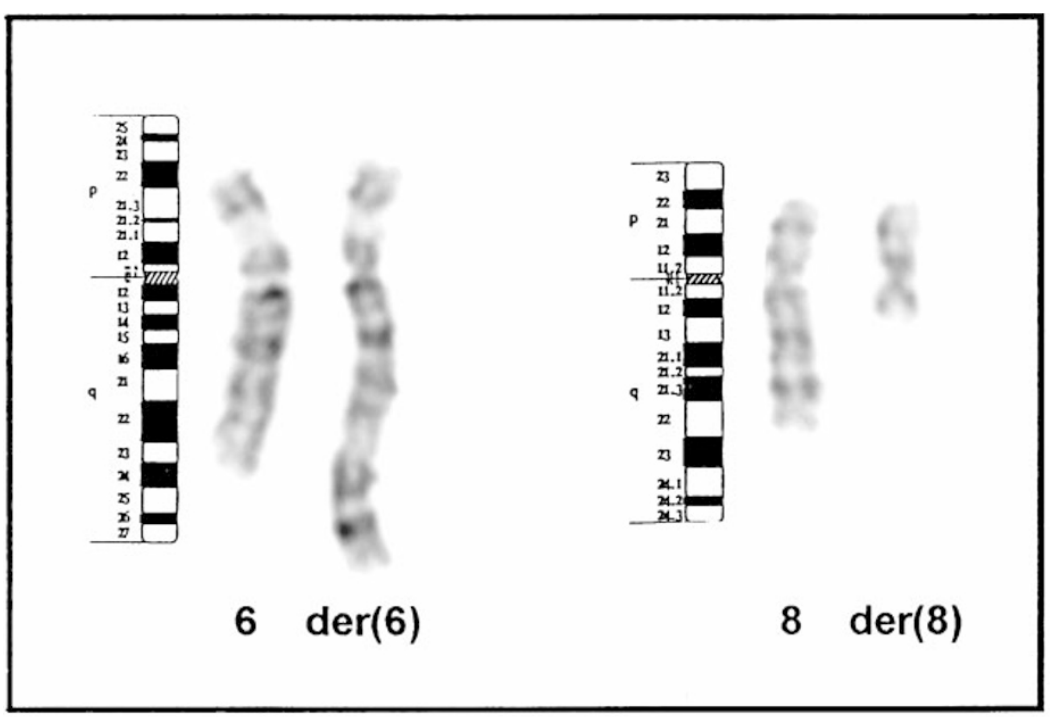

a

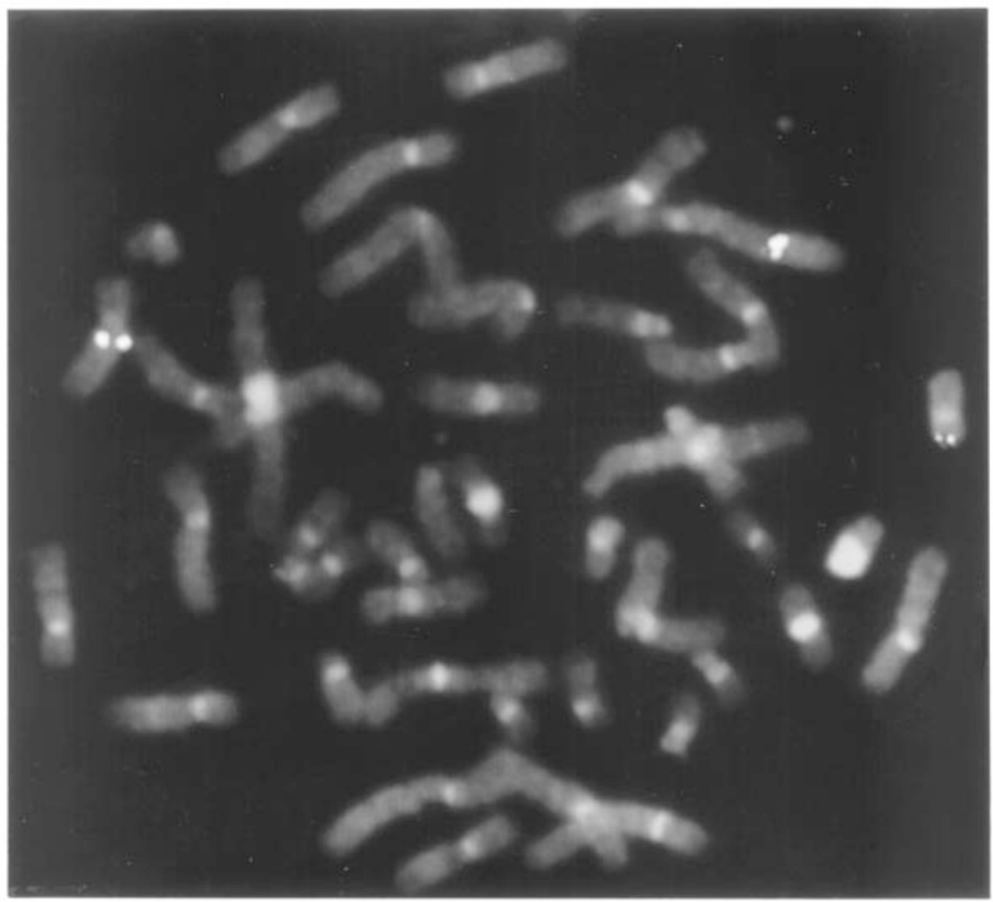

b

Figure 1 a Partial karyotype of Duane patient showing a t(6;8)(q26;q13); b FISH analysis with YAC clone820E6 shows a large signal on normal chromosome8, one signal on der(8) and one signal on der(6) as for bridging the 8q13 breakpoint.

was also confirmed by STS content analysis of microdissected DNA from der(6) and der(8). To refine the SRO map, we have isolated two PAC clones positive for markers WI4901 and SHGC37325, respectively. SHGC37325 maps distal to AFM238xc3 and proximal to WI4901. ${ }^{11}$ By FISH analysis these clones were found to border the 8q13 breakpoint. Thus, the breakpoint maps between the two clones in a segment estimated to be approximately $40 \mathrm{~kb}$, as measured fibre-FISH (although the involvement of PAC ends flanking this region cannot be excluded).

At present no gene has been assigned to this Duane syndrome gene interval. ${ }^{11}$ Based on the possible neuromuscular origin of Duane syndrome and the gonadal dysgenesis present in the patient, an electronic search for genes at the 


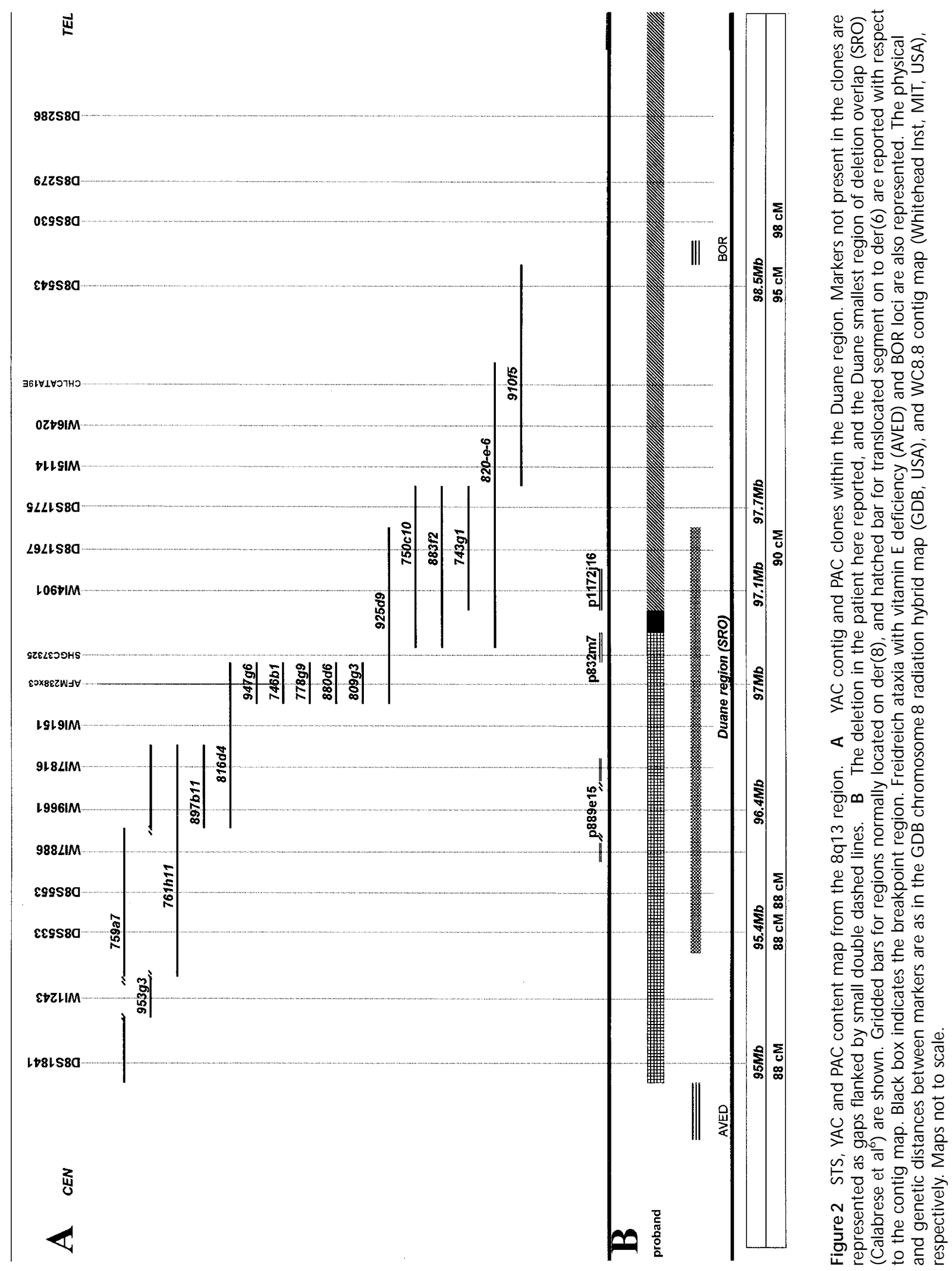



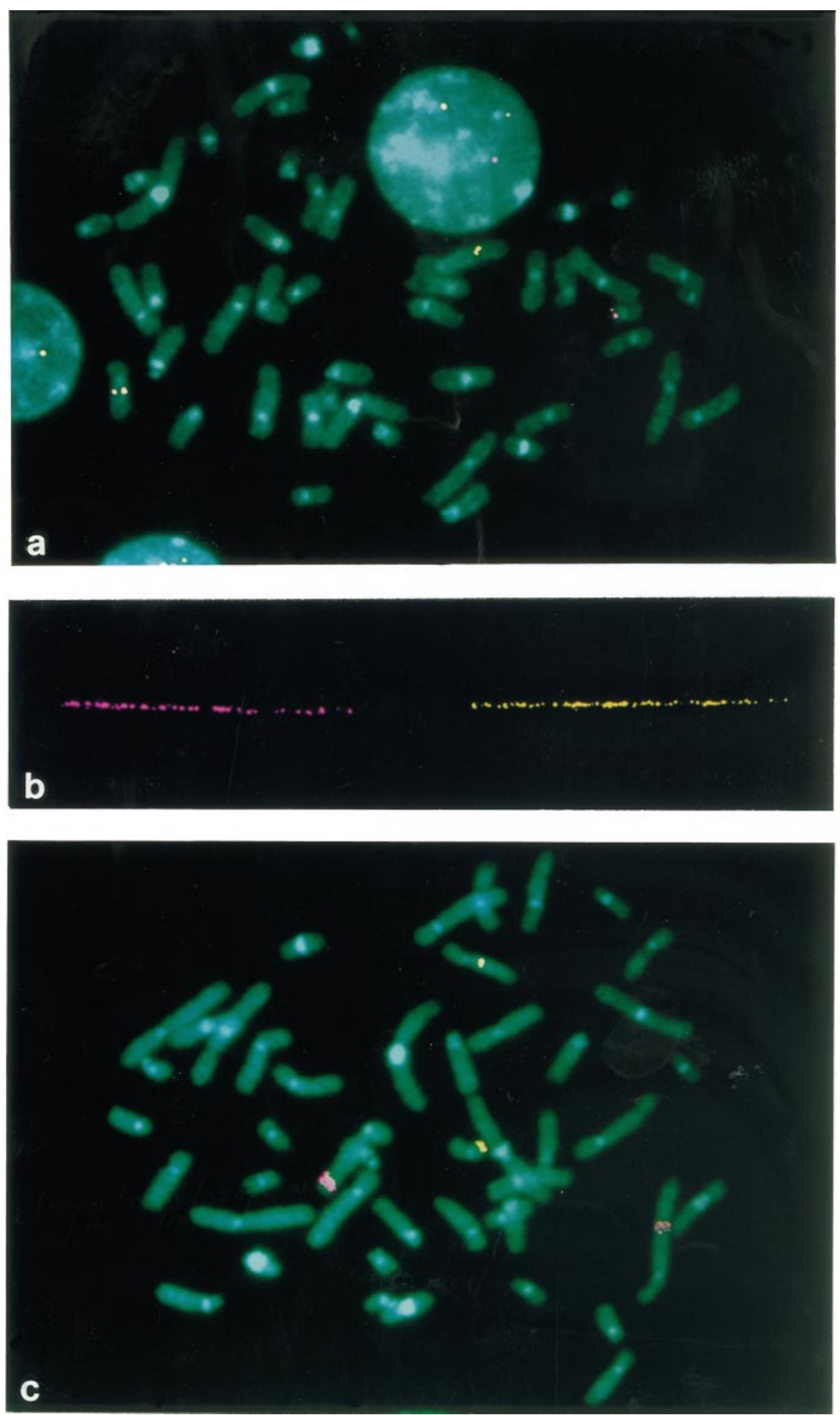

Figure 3 a Dual color FISH using PAC clones832m7 and 1172j16 selected with markers SHGC37325 and WI4901, respectively. Clone 832m7 shows signals on der(8) (red), while clone 1172j16 shows signals on der(6) (yellow-green). On the normal chromosome 8, signals from the two probes result in an orange-yellow spot due to combined yellow-green and red fluorescences; b fibre-FISH showing PAC clones $832 \mathrm{m7}$ (red array) and 1172j16 (yellow-green array) as separated by a $40 \mathrm{~kb}$ gap on the basis of the insert length of the two clones ( $120 \mathrm{~kb}$ each); c FISH with PAC clone $889 \mathrm{e} 15$ displaying yellow spots on normal chromosome 8 at band q13 and on der(8). A control 6q25 specific painting probe is also shown in red. 
8q12-q21 region suggested $A M Y B$ as a putative candidate gene because of its expression in hindbrain, neural retina and genital crests. ${ }^{7}$ Two ESTs, WI7886 and WI7816 containing 5' and 3'UTR AMYB sequences, mapped with in the Duane SRO, suggested that the site for AMYB was more proximal, at $8 q 13$, than previously reported $8 q 21-q 22$. This was demonstrated by FISH using PAC clone889el5 positive with the two ESTs which showed signals on $\operatorname{der}(8)$ only. Therefore, our data map the AMYB locus at least $300 \mathrm{~kb}$ proximal to the $8 \mathrm{q}$ breakpoint in the patient and exclude AMYB as a Duane syndrome candidate gene.

In conclusion, the association of Duane syndrome with a $8 q 13$ rearrangement in the patient described here and in two previously reported cases ${ }^{5,6}$ strongly supports the location of a gene for Duane syndrome at $8 q 13$. In this study the $8 q$ Duane syndrome gene interval has been narrowed down to a $40 \mathrm{~kb}$ interval between markers SHGC37325 and WI4901. These results will greatly facilitate the identification of the causative gene.

\section{Acknowledgements}

The authors wish to thank C Sala and M Mancini at IGeR, Milan, for providing the YAC and PAC clones. The financial support of Telethon-Italy (grantE689) is gratefully acknowledged.

\section{References}

1 Online Mendelian Inheritance in man (OMIM): http://www .ncbi.nIm.gov/OMIM.

2 Bateman JB, Isenberg SJ: Genetic aspects of strabismus. In: Emery AEH, Rimoin DL (eds). Principles and Practice of Medical Genetics, 2nd edn. Edinburgh: Churchill Livingstone, 1990, pp 726-727.
3 Huber A: Electrophysiology of the retraction syndrome. $\mathrm{Br} \mathrm{J}$ Ophthalmol 1974; 58: 293-300.

4 Ott S, Borchert M, Chung M et al: Exclusion of candidate genetic loci for Duane retraction syndrome. Am J Ophthalmol 1999; 127: 358-360.

5 Vincent C, Kalatzis V, Compain S et al: A proposed new contiguous gene syndrome on 8q consists of Branchio-Oto-Renal (BOR) syndrome, Duane syndrome, a dominant form of hydrocephalus and trapeze aplasia; implications for the mapping of the BOR gene. Hum Mol Genet 1994; 3: 1859-1866.

6 Calabrese G, Stuppia L, M orizio E et al: Detection of an insertiondeletion of region 8q13-q21.2 in a patient with Duane syndrome: implications for mapping and cloning of a Duane gene. Eur J Hum Genet 1998; 6: 187-193.

7 Trauth K, Mutschler B, Jenkins NA, Cilbert DJ, Copeland NG, Klempnauer $\mathrm{KH}$ : Mouse A-myb encodes a trans-activator and is expressed in mitotically active cells of the developing central nervous system, adult testis and B lymphocytes. EM BO J 1994; 13: 5994-6005.

8 Fidlerova H, Senger G, Kost M, Sanseau P, Sheer D: Two simple procedures for releasing chromatin from routinely cells for fluorescence in situ hybridization. Cytogenet Cell Genet 1994; 65: 203-205.

9 Chumakov IM, Rigault P, Le Gall I et al: A YAC contig of the human genome. Nature 1995; 377 suppl.: 175-299.

10 Whitehead Institute for Biomedical Research/MIT Center for Human Research: http://www-genome.wi.mit.edu.

11 Genome Data Base: http://www.gdb.org.

12 Zhang J, Meltzer P, Trent JM: Rapid isolation and characterization of amplified DNA by chromosome microdissection: identification of IGF1R amplification in malignant melanoma. Oncogene 1993; 8: 2827-2832.

13 Telenius H, Palmear AH, Tunnacliffe A et al: Cytogenetic analysis by chromosome painting using DOP-PCR amplified flow-sorted chromosomes. Genes Chrom Cancer 1992; 4: 257-263. 